\title{
Effects of a luteinizing hormone-releasing hormone agonist on cognitive, sexual, and hormonal functions in patients with prostate cancer: relationship with testicular and adrenal androgen levels
}

Kohei Okamoto, Yositaka Sekine, Masashi Nomura, Hidekazu Koike, Hiroshi Matsui, Yasuhiro Shibata, Kazuto Ito and Kazuhiro Suzuki

\begin{abstract}
Purpose: To assess the cognitive and sexual/hormonal functioning of prostate cancer patients treated with a luteinizing hormone-releasing hormone $(\mathrm{LH}-\mathrm{RH})$ agonist, and the relationships thereof with adrenal and residual testicular androgen levels.

Materials and methods: Previously, we reported the effect of a luteinizing hormone-releasing hormone ( $\mathrm{LH}-\mathrm{RH})$ agonist on testicular and adrenal androgen production in patients with prostate cancer. A 6-month treatment with an LH-RH agonist significantly reduced testicular androgens by $90-95 \%$ and adrenal androgens by $26-40 \%$. This study evaluated the changes in cognitive and sexual/hormonal functions in the same cohort using the Mini-Mental State Evaluation (MMSE) and Expanded Prostate Cancer Index Composite (EPIC) questionnaire, respectively. In addition, the associations of each function with the serum testosterone (T), dihydrotestosterone (DHT), estradiol (E2), dehydroepiandrosterone-sulfate (DHEA-S), dehydroepiandrosterone (DHEA), androstenedione (A-dione), and cortisol levels were studied.

Results: Cognitive functions did not change significantly during the treatment. Sexual functions were relatively low before treatment and worsened significantly after 6 and 12 months of treatment. Interestingly, sexual bothers were improved with the treatment. The treatment significantly worsened hormonal functions and bothers. Regarding specific items in the hormonal domains, hot flashes and body weight changes were the main effects of worsened hormonal function. Low levels of T and E2 and high levels of A-dione were associated with low MMSE scores at 6 months. Regarding sexual and hormonal functions, A-dione, E2, T, cortisol, and DHEA-S were associated with poorer functioning and bother. Especially, low T levels and high E2 levels were the most significant factors associated with worse sexual and hormonal bothers.

Conclusion: The LH-RH agonist monotherapy worsened sexual and hormonal functions and hormonal bothers, but not sexual bothers or cognitive functions. The changes in these functions were related to the testicular and adrenal androgens levels.
\end{abstract}

Keywords: Prostate cancer, LH-RH agonist, Sexual and hormone functions, Cognitive function, Androgens

\footnotetext{
* Correspondence: kazu@gunma-u.ac.jp

Department of Urology, Gunma University Graduate School of Medicine,

3-39-22 Showa-machi, Maebashi 371-8511, Gunma, Japan
} 


\section{Introduction}

Prostate cancer is an androgen-dependent cancer. Longterm androgen-deprivation therapy (ADT) is the standard therapy for metastatic prostate cancer and is administered in localized or locally progressive disease concomitant with radiation therapy for the short or long term [1]. ADT has several adverse effects [2,3]. ADT with luteinizing hormone-releasing hormone (LH-RH) analogues is used frequently for medical castration. Previously, we reported the effect of an LH-RH agonist on reducing serum adrenal androgen levels 6 and 12 months after initiating treatment [4]. We also identified immunoreactive LH receptors in the reticular layer of the adrenal glands and speculated that the LH-RH agonist therapy reduced adrenal androgen synthesis via reduced LH levels [4].

This study assessed the adverse effects of ADT in the same cohort, focusing on cognitive and sexual/hormone functions. We evaluated the changes in the healthrelated quality of life (QOL) and cognitive functions using the Expanded Prostate Cancer Index Composite (EPIC) questionnaire [5,6] and Mini-Mental State Evaluation (MMSE) [7]. In addition, the changes in the QOL and cognitive function parameters were compared with serum levels of testicular and adrenal androgens.

\section{Materials and methods}

\section{Patients}

Previously, we evaluated the serum testicular and adrenal androgen levels in 47 patients with prostate cancer treated via 6-month neoadjuvant $\mathrm{ADT}$ with radiation therapy, followed by adjuvant ADT (this treatment strategy was reported previously by our study group [8]). ADT was given as monotherapy; we prescribed the LHRH agonist leuprolide. Forty-five of the forty-seven patients had full QOL and cognitive function test data and were included in this study. Table 1 shows the clinical characteristics of the patients analyzed. Their ages ranged from 61 to 75 years, with a mean age of 67.5 years. The mean pretreatment prostate-specific antigen (PSA) level was $13.0 \mathrm{ng} / \mathrm{mL}$. Using the D'Amico risk stratification, 16 patients $(35.6 \%)$ were categorized in the intermediate-risk group and 29 patients (64.4\%) in the high-risk group. This study was approved by the Ethics Committee of the Gunma University Faculty of Medicine, and written consent was obtained from the enrolled patients.

\section{QOL questionnaires and cognitive function test}

The QOL was assessed using the EPIC questionnaire [5] before and 6 and 12 months after initiating the LH-RH agonist treatment. In this study, the sexual and hormonal domains were analyzed. For the sexual domain, the mean summary scores and subscale scores; i.e., sexual function and bother, were assessed. For the hormonal domain, the score changes for all items in the hormonal
Table 1 Clinical characteristics of the enrolled patients

\begin{tabular}{lll}
\hline $\mathrm{n}$ & 45 & \\
Age $( \pm$ SD $)$ & $67.5 \pm 3.5$ & \\
PSA $( \pm$ SD $)$ & $13 \pm 10.7$ & 1 \\
G.S. & 6 & 28 \\
& 7 & 9 \\
& 8 & 7 \\
T & 9 & 12 \\
& T1c & 1 \\
& T2a & 7 \\
& T2b & 7 \\
& T2c & 17 \\
Risk group & T3a & 1 \\
& T3b & 16 \\
& Intermediate & 29 \\
\hline
\end{tabular}

PSA: prostate specific antigen.

G.S. Gleason score.

T:T category of TNM Classification of Malignant Tumours 7th Edition.

Risk group: D'Amico risk stratification.

domain were studied, as well as the mean summary and subscale scores. Statistical significance was determined using the $x^{2}$ test. Cognitive function was assessed using Mini-Metal State Examination [7] and the same time points as the QOL assessment. The examination was administered by one medical technologist (R.S.).

\section{Blood samples and measuring hormone levels}

As described elsewhere [4], serum testosterone (T), dihydrotestosterone (DHT), estradiol (E2), dehydroepiandrosterone (DHEA), and androstenedione (A-dione) were measured using liquid chromatography-mass spectrometry (LC-MS). DHEA-sulfate (DHEA-S) was measured using a chemiluminescence enzyme immunity assay. In this study, we also measured the serum cortisol levels using LC-MS. Blood samples were taken before and 6 and 12 months after initiating the LH-RH treatment. The changes in hormone levels at each evaluation point are shown in Table 2. As mentioned earlier, the serum levels of T, DHT, E2, DHEA-S, DHEA, and A-dione decreased significantly during the treatment, while the cortisol levels did not differ significantly.

\section{Evaluation of sexual, hormonal, and cognitive functions according to age-adjusted hormone levels}

To evaluate the association between the hormonal environments and QOL and MMSE scores at each observation point, a multivariate analysis was performed. As dependent variables, the MMSE scores, and sexual and hormonal subscale scores were adopted $(0 \geq$ mean, $1<$ mean). Differential values were the hormone levels and age at each observation 
Table 2 Changes in the serum hormone levels in prostate cancer patients treated with a luteinizing hormone-releasing hormone agonist

\begin{tabular}{|c|c|c|c|c|c|}
\hline & & PRE & $6 \mathrm{MO}$ & $12 \mathrm{MO}$ & Statistics \\
\hline \multirow[t]{2}{*}{ T } & Mean (ng/dL) & $378.8 \pm 145.1$ & $9.6 \pm 5.0$ & $7.7 \pm 4.6$ & $P<0.01$ PRE Vs, $12 \mathrm{MO}$ \\
\hline & Percentile change & & $-97.5 \%$ & $-98.0 \%$ & \\
\hline \multirow[t]{2}{*}{ DHT } & Mean $(\mathrm{pg} / \mathrm{mL})$ & $420.2 \pm 200.2$ & $21.6 \pm 12.2$ & $20.8 \pm 11.2$ & $\mathrm{P}<0.01$ PRE vs $6 \mathrm{MO}, 12 \mathrm{MO}$ \\
\hline & Percentile change & & $-94.9 \%$ & $-95.0 \%$ & \\
\hline \multirow[t]{2}{*}{ E2 } & Mean $(\mathrm{pg} / \mathrm{mL})$ & $16.2 \pm 5.2$ & $1.3 \pm 1.0$ & $1.4 \pm 1.0$ & $\mathrm{p}<0.01$ PRE vs $6 \mathrm{MO}, 12 \mathrm{MO}$ \\
\hline & Percentile change & & $-92.2 \%$ & $-91.3 \%$ & \\
\hline \multirow[t]{2}{*}{ DHEA-S } & Mean $(\mu \mathrm{g} / \mathrm{dL})$ & $144.9 \pm 66.6$ & $107.2 \pm 48.6$ & $103.5 \pm 54.3$ & $\mathrm{P}<0.01$ PRE vs $6 \mathrm{MO}, 12 \mathrm{MO}$ \\
\hline & Percentile change & & $-26.1 \%$ & $-28.6 \%$ & \\
\hline \multirow[t]{2}{*}{ DHEA } & Mean (ng/mL) & $1.85 \pm 0.91$ & $1.38 \pm 0.67$ & $1.29 \pm 0.65$ & $\mathrm{P}<0.01$ PRE vs $6 \mathrm{MO}, 12 \mathrm{MO}$ \\
\hline & Percentile change & & $-25.1 \%$ & $-30.0 \%$ & \\
\hline \multirow[t]{2}{*}{ A-dione } & Mean (pg/mL) & $384.0 \pm 133.5$ & $231.7 \pm 109.4$ & $227.8 \pm 119.7$ & $\mathrm{p}<0.01$ PRE vs $6 \mathrm{MO}, 12 \mathrm{MO}$ \\
\hline & Percentile change & & $-39.7 \%$ & $-40.7 \%$ & \\
\hline \multirow[t]{2}{*}{ cortisol } & Mean (ng/mL) & $89.9 \pm 26.7$ & $94.9 \pm 27.4$ & $81.4 \pm 23.4$ & Not significant \\
\hline & Percentile change & & $5.4 \%$ & $-10.4 \%$ & \\
\hline
\end{tabular}

PRE: pretreatment 6MO: 6 months after initiation of LH-RH agonist treatment.

12MO: 12 months after initiation of LH-RH agonist treatment.

T: testosterone, DHT: dihydrotestosterone, E2; estradiol, DHEA-S: dehydroepiandrosterone-sulfate.

DHEA: dehydroepiandrosterone.

Percentile change: changes in comparison with pretreatment levels.

The percentile change is in comparison with the pretreatment levels. Values are expressed as means \pm SD (standard deviation).

point. Using stepwise multiple regression analysis, significant predictive factors were estimated. The statistical analysis was performed using SPSS ver. 19.0 (SPSS, Chicago, IL, USA).

\section{Results}

The changes in the MMSE scores are shown in Figure 1. The mean pretreatment score was 28.7 , and no significant difference was observed after 6 or 12 months of treatment. Stratification of the patients based on pretreatment scores; i.e., $\geq$ mean or $<$ mean, showed no significant difference in the mean scores during treatment

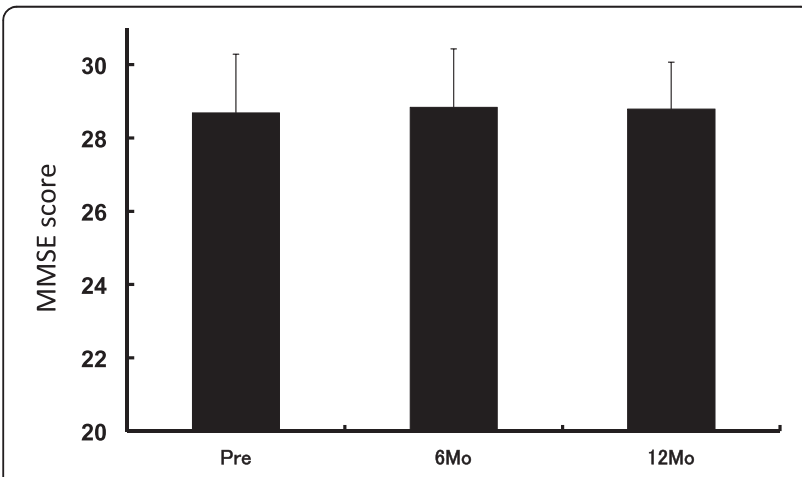

Figure 1 Changes in MMSE scores. Values are expressed as means \pm SD. PRE: pretreatment, $6 \mathrm{Mo}$ : 6 months after initiation of treatment, 12Mo: 12 months after initiation of treatment. in either the high or low baseline score groups (data not shown).

Figure 2 shows the changes in the sexual and hormonal scores. The summary scores of both the sexual and hormonal domains worsened significantly during treatment. Regarding the subscale scores, the sexual function scores worsened significantly, whereas the sexual bother scores improved significantly. By contrast, the scores for both hormonal function and bother worsened significantly.

Tables 3 and 4 summarize the frequency of each item for hormonal function and bother, respectively. The functional and bother changes; i.e., hot flashes, were worsened significantly during treatment. Significant body weight gains were observed.

Finally, we studied the associations of cognitive function, sexual, and age-adjusted hormonal functions with serum hormone levels. Low MMSE scores were associated with low E2 and cortisol and high A-dione levels at 6 months. None of the pretreatment hormone levels or decreased hormone levels were significantly related to the scores during the treatment. Regarding the sexual and hormonal function and bother scores, A-dione, E2, $\mathrm{T}$, cortisol, and DHEA-S were associated with poorer functioning and bother at each observation point, as shown in Table 5. Low $\mathrm{T}$ levels were the factors most significantly associated with worsened sexual and hormonal bother at 6 months. 


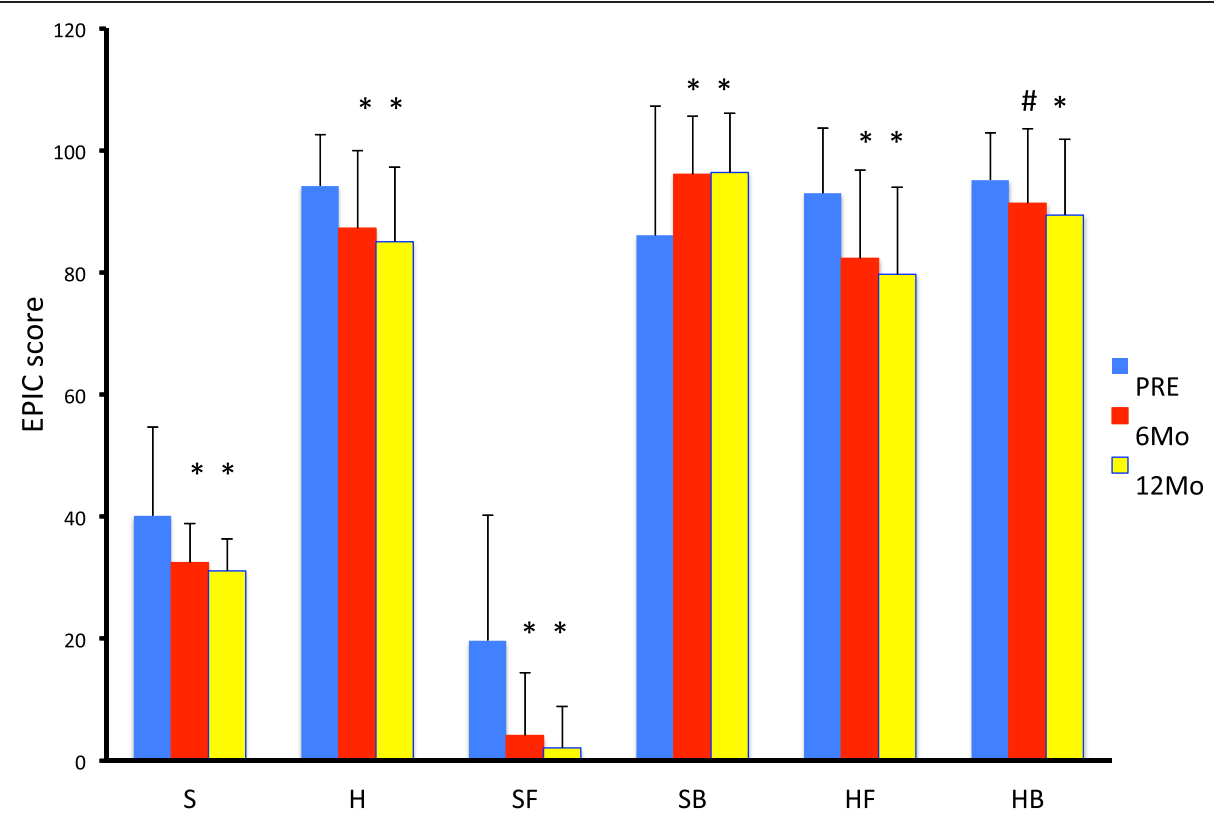

Figure 2 Mean sexual and hormone domain scores including summary score and subscale scores. Values are expressed as means \pm SD, and pretreatment values are compared with those at each post-treatment sampling point. A difference was considered to be significant if the $p$-value was less than 0.05 ( \# $p<0.05,{ }^{*} p<0.01$ ). EPIC: Expanded Prostate Cancer Index Composite. PRE: pretreatment, 6 Mo: 6 months after initiation of treatment, $12 \mathrm{Mo}: 12$ months after initiation of treatment Summary score: S; sexual summary score including sexual function and sexual bother scores. H; hormone summary score including hormone function and hormone bother scores. Subscale scores: SF; sexual function, $\mathrm{SB}$; sexual bother, HF; hormonal function, HB; hormone bother.

Table 3 Distribution of EPIC hormone function domain items before and after 6 and 12 months of androgen-deprivation therapy

\begin{tabular}{|c|c|c|c|c|c|}
\hline & More than once a day & About once a day & More than once a week & About once a week & Rarely or never \\
\hline \multicolumn{6}{|c|}{ Hot flashes* } \\
\hline Pre & $4.4 \%$ & $4.4 \%$ & $0.0 \%$ & $6.7 \%$ & $84.4 \%$ \\
\hline $6 \mathrm{Mo}$ & $33.3 \%$ & $13.3 \%$ & $6.7 \%$ & $0.0 \%$ & $46.7 \%$ \\
\hline $12 \mathrm{Mo}$ & $44.4 \%$ & $8.9 \%$ & $2.2 \%$ & $4.4 \%$ & $40.0 \%$ \\
\hline \multicolumn{6}{|c|}{ Breast tenderness } \\
\hline Pre & $2.2 \%$ & $0.0 \%$ & $0.0 \%$ & $0.0 \%$ & $97.8 \%$ \\
\hline $6 \mathrm{Mo}$ & $0.0 \%$ & $2.2 \%$ & $0.0 \%$ & $0.0 \%$ & $97.8 \%$ \\
\hline $12 \mathrm{Mo}$ & $0.0 \%$ & $0.0 \%$ & $2.2 \%$ & $2.2 \%$ & $95.6 \%$ \\
\hline \multicolumn{6}{|c|}{ Feel depressed } \\
\hline Pre & $0.0 \%$ & $2.2 \%$ & $2.2 \%$ & $13.3 \%$ & $82.2 \%$ \\
\hline $6 \mathrm{Mo}$ & $0.0 \%$ & $2.2 \%$ & $4.4 \%$ & $8.9 \%$ & $84.4 \%$ \\
\hline $12 \mathrm{Mo}$ & $0.0 \%$ & $0.0 \%$ & $4.4 \%$ & $13.3 \%$ & $82.2 \%$ \\
\hline \multicolumn{6}{|c|}{ Lack of energy } \\
\hline Pre & $0.0 \%$ & $6.7 \%$ & $6.7 \%$ & $22.2 \%$ & $64.4 \%$ \\
\hline $6 \mathrm{Mo}$ & $0.0 \%$ & $4.4 \%$ & $11.1 \%$ & $15.6 \%$ & $68.9 \%$ \\
\hline \multirow[t]{2}{*}{$12 \mathrm{Mo}$} & $2.2 \%$ & $6.7 \%$ & $15.6 \%$ & $15.6 \%$ & $60.0 \%$ \\
\hline & Gained $5 \mathrm{~kg}$ or more & Gained less than 5 kg & No change & Lost less than $5 \mathrm{~kg}$ & Lost $5 \mathrm{~kg}$ or more \\
\hline \multicolumn{6}{|c|}{ Change in body weight* } \\
\hline Pre & $0.0 \%$ & $2.2 \%$ & $93.3 \%$ & $4.4 \%$ & $0.0 \%$ \\
\hline $6 \mathrm{Mo}$ & $2.2 \%$ & $31.1 \%$ & $60.0 \%$ & $6.7 \%$ & $0.0 \%$ \\
\hline $12 \mathrm{Mo}$ & $2.2 \%$ & $35.6 \%$ & $57.8 \%$ & $4.4 \%$ & $0.0 \%$ \\
\hline
\end{tabular}

${ }^{*} \mathrm{p}<0.001$. 
Table 4 Distribution of the EPIC hormone bother domain items before and after 6 and 12 months of androgen-deprivation therapy

\begin{tabular}{|c|c|c|c|c|c|c|}
\hline & No such symptom & No problem & Very small problem & Small problem & Moderate problem & Big problem \\
\hline \multicolumn{7}{|c|}{ Hot flashes* } \\
\hline Pre & $80.0 \%$ & $6.7 \%$ & $11.1 \%$ & $2.2 \%$ & $0.0 \%$ & $0.0 \%$ \\
\hline $6 \mathrm{Mo}$ & $46.7 \%$ & $11.1 \%$ & $20.0 \%$ & $8.9 \%$ & $13.3 \%$ & $0.0 \%$ \\
\hline $12 \mathrm{Mo}$ & $31.1 \%$ & $15.6 \%$ & $17.8 \%$ & $20.0 \%$ & $8.9 \%$ & $6.7 \%$ \\
\hline \multicolumn{7}{|c|}{ Breast tenderness/enlargement } \\
\hline Pre & $95.6 \%$ & $2.2 \%$ & $0.0 \%$ & $0.0 \%$ & $0.0 \%$ & $2.2 \%$ \\
\hline $6 \mathrm{Mo}$ & $93.3 \%$ & $4.4 \%$ & $2.2 \%$ & $0.0 \%$ & $0.0 \%$ & $0.0 \%$ \\
\hline $12 \mathrm{Mo}$ & $84.4 \%$ & $8.9 \%$ & $4.4 \%$ & $2.2 \%$ & $0.0 \%$ & $0.0 \%$ \\
\hline \multicolumn{7}{|c|}{ Loss of body hair } \\
\hline Pre & $91.1 \%$ & $6.7 \%$ & $0.0 \%$ & $0.0 \%$ & $2.2 \%$ & $0.0 \%$ \\
\hline $6 \mathrm{Mo}$ & $86.7 \%$ & $6.7 \%$ & $6.7 \%$ & $0.0 \%$ & $0.0 \%$ & $0.0 \%$ \\
\hline $12 \mathrm{Mo}$ & $66.7 \%$ & $15.6 \%$ & $8.9 \%$ & $6.7 \%$ & $2.2 \%$ & $0.0 \%$ \\
\hline \multicolumn{7}{|c|}{ Feeling depressed } \\
\hline Pre & $66.7 \%$ & $8.9 \%$ & $17.8 \%$ & $4.4 \%$ & $2.2 \%$ & $0.0 \%$ \\
\hline $6 \mathrm{Mo}$ & $62.2 \%$ & $17.8 \%$ & $8.9 \%$ & $6.7 \%$ & $4.4 \%$ & $0.0 \%$ \\
\hline $12 \mathrm{Mo}$ & $66.7 \%$ & $17.8 \%$ & $8.9 \%$ & $6.7 \%$ & $0.0 \%$ & $0.0 \%$ \\
\hline \multicolumn{7}{|c|}{ Lack of energy } \\
\hline Pre & $57.8 \%$ & $11.1 \%$ & $15.6 \%$ & $15.6 \%$ & $0.0 \%$ & $0.0 \%$ \\
\hline $6 \mathrm{Mo}$ & $55.6 \%$ & $17.8 \%$ & $13.3 \%$ & $8.9 \%$ & $4.4 \%$ & $0.0 \%$ \\
\hline $12 \mathrm{Mo}$ & $46.7 \%$ & $15.6 \%$ & $28.9 \%$ & $4.4 \%$ & $2.2 \%$ & $2.2 \%$ \\
\hline \multicolumn{7}{|c|}{ Change in body weight } \\
\hline Pre & $84.4 \%$ & $11.1 \%$ & $4.4 \%$ & $0.0 \%$ & $0.0 \%$ & $0.0 \%$ \\
\hline $6 \mathrm{Mo}$ & $57.8 \%$ & $17.8 \%$ & $15.6 \%$ & $4.4 \%$ & $2.2 \%$ & $2.2 \%$ \\
\hline $12 \mathrm{Mo}$ & $62.2 \%$ & $20.0 \%$ & $11.1 \%$ & $4.4 \%$ & $0.0 \%$ & $2.2 \%$ \\
\hline
\end{tabular}

\section{Discussion}

This study examined the changes in cognitive function using the MMSE and in sexual and hormonal functions using the EPIC questionnaire.

We observed no significant worsening in the MMSE scores in this study. The effects of ADT on cognitive functions are controversial. Nelson et al. [9] summarized nine studies on this matter, and stated that ADT impaired cognitive functions subtly, especially the visuospatial abilities and executive functioning. Alibhai et al. [10] compared the cognitive functions of non-metastatic prostate cancer patients treated with ADT with those of prostate cancer patients not receiving ADT and healthy controls. They observed no consistent evidence of adverse effects on cognitive functions. In a similar study, Mohile et al. [11] focused on the preexisting impairment of cognitive functions in elderly subjects and stated that the baseline prevalence of cognitive impairment affected the results. We stratified the patients according to the baseline MMSE scores and found no significant difference during the study. Recently, Chao et al. [12] reported a prospective study of the effects of ADT on brain function using functional MRI. In that study, 6 months of ADT clearly impaired brain activations during cognitive control and functional brain connectivity on functional MRI. Interestingly, cognitive function tests showed no significant impairment at this point. Further studies of the association of conventional cognitive function tests with brain function imaging are warranted.

The QOL was assessed using the EPIC questionnaire, which has been validated in Japanese [5]. The summary scores of both sexual and hormonal functions at 6 and 12 months worsened significantly in comparison with those before treatment. Although the sexual functions worsened significantly while on ADT, sexual bother improved during the treatment. This tendency was consistent with the results of the validation study [5]. The exact reason remains unknown; however, the loss of libido might reduce the sexual desire that causes sexual bother. The trends in the hormonal function and bother scores were similar to and consistent with the validation study [5]. 
Table 5 Association between hormone levels and the MMSE and EPIC scores: multivariate analysis

\begin{tabular}{|c|c|c|c|c|c|}
\hline \multicolumn{6}{|l|}{ MMSE } \\
\hline \multirow[t]{4}{*}{6 Mo hormone levels } & & B & Odds ratio & $95 \% \mathrm{Cl}$ & $\mathrm{p}$ \\
\hline & E2 & -1.47 & 0.23 & $0.073-0.724$ & 0.012 \\
\hline & A-dione & 0.021 & 1.02 & $1.006-1.036$ & 0.006 \\
\hline & cortisol & -0.059 & 0.94 & $0.898-0.989$ & 0.016 \\
\hline \multicolumn{6}{|l|}{ EPIC } \\
\hline \multicolumn{6}{|c|}{ Hormone levels and QOL at each observation point } \\
\hline \multicolumn{6}{|l|}{ PRE } \\
\hline Sexual bother & A-dione & -0.02 & 0.98 & $0.964-0.997$ & 0.019 \\
\hline \multicolumn{6}{|l|}{$6 \mathrm{Mo}$} \\
\hline \multirow[t]{3}{*}{ Sexual bother } & E2 & 1.57 & 4.81 & $1.086-21.33$ & 0.039 \\
\hline & $\mathrm{T}$ & -61.15 & $2.76 \times 10^{-27}$ & $1.34 \times 10^{-5}-0.00057$ & 0.026 \\
\hline & DHT & 0.21 & 1.017 & $1.028-1.484$ & 0.024 \\
\hline \multirow[t]{2}{*}{ Hormonal function } & E2 & 1.14 & 3.12 & $1.13-8.64$ & 0.028 \\
\hline & A-dione & -0.019 & 0.98 & $0.97-0.998$ & 0.026 \\
\hline \multirow[t]{3}{*}{ Hormonal bother } & E2 & 2.006 & 7.44 & $1.82-30.30$ & 0.005 \\
\hline & $\mathrm{T}$ & -30.8 & $4.19 \times 10^{-14}$ & $4.32 \times 10^{-24}-0.00041$ & 0.017 \\
\hline & cortisol & 0.076 & 1.08 & $1.02-1.14$ & 0.008 \\
\hline \multicolumn{6}{|l|}{$12 \mathrm{Mo}$} \\
\hline Sexual bother & E2 & 1.082 & 2.95 & $1.11-7.82$ & 0.030 \\
\hline \multirow[t]{2}{*}{ Hormonal bother } & A-dione & -0.0089 & 0.991 & 0.983-0.9997 & 0.042 \\
\hline & DHEA-S & -0.0174 & 0.983 & 0.966-0.999 & 0.049 \\
\hline
\end{tabular}

B: regression coefficient.

PRE: pretreatment 6MO: 6 months after initiation of LH-RH agonist treatment.

12MO: 12 months after initiation of LH-RH agonist treatment.

T: testosterone, DHT: dihydrotestosterone, E2; estradiol, DHEA-S: dehydroepiandrosterone-sulfate.

A-dione: androstenedion, MMSE: mini-mental state examination.

EPIC: expanded postate cancer index composite.

In this study, we focused on the hormone levels and QOL assessments and showed the detailed changes in the hormonal domain scores (Tables 3 and 4). Among hormonal functions, hot flashes and body weight gain were worsened significantly on ADT. Only hot flashes were significantly associated with bother. Body weight gain is one of the important adverse events caused by ADT. In the present study, about one-third of all patients experienced body weight gain after 6 months of ADT. Interestingly, the proportions of patients experiencing body weight gains $>5 \mathrm{~kg}$ or $\leq 5 \mathrm{~kg}$ were very similar at 6 and 12 months. Lee et al. [13] studied the effect of ADT on body composition changes in prostate cancer patients. On $\mathrm{ADT}$, the fat and lean masses were increased significantly only in patients not receiving ADT. By contrast, a significant loss in bone mineral density occurred in both the patients not receiving ADT or those pretreated with ADT. Our findings regarding body weight changes are consistent with these findings. This information would be helpful when obtaining informed consent from patients who need ADT. Gay et al. reported on the QOL assessment using the EPIC questionnaire in patients with prostate cancer treated with neoadjuvant ADT [14]. The sexual and hormonal summary scores were decreased significantly after 2 months of neoadjuvant ADT. In this study, the question items for hormone bother were summarized. The percentage of patients with bother tended to be higher in the cohort of Gay et al. [14] in comparison with our study. This might be due to age differences, 70.2 vs. 67.5 years, ethnic differences, or tolerance of ADT.

Finally, we investigated the association of cognitive, sexual, and hormone functions with serum hormone levels. In a previous study, we detected a significant decrease in both testicular and adrenal androgens after LH-RH agonist treatment [4]. The decreases in T and E2 were associated with cognitive functions [15]. However, the association of adrenal androgen levels with cognitive or sexual/hormone functions has not been examined. Furthermore, cortisol levels and stress are related [16], so we also examined the cortisol levels. In the cognitive function test, low E2 levels were significantly related to worse MMSE scores in our study. This finding was consistent with Salminen et al. [17], who investigated 
cognitive functions in 23 patients with ADT-treated prostate cancer. The serum E2 levels before and after 6 and 12 months of treatment were correlated with visual memory and verbal fluency. In our study, the A-dione and cortisol levels were associated with the MMSE scores. The odds ratios of A-dione and cortisol were 1.02 and 0.94 , respectively, and the role of hormonal changes was unknown. Regarding sexual and hormone functions, low $\mathrm{T}$ levels were the factor most significantly associated with worse sexual and hormone bother. High E2 levels were significantly associated with worse sexual bother and both hormone function and bother. In males, $\mathrm{E} 2$ is synthesized from $\mathrm{T}$ by aromatization in peripheral tissues, including fat [18]. ADT causes a change in body composition, and the fat mass generally increases [13]. In our series, $33.3 \%$ of the patients experienced significant body weight gain during the first 6 months of ADT. High E2 levels might affect the QOL changes via this mechanism. High cortisol levels were associated with worse hormonal bother. Cortisol is the most researched stress hormone [16]. Stress during ADT might be associated with high cortisol levels. We observed that low adrenal androgen A-dione and DHEA-S levels were significantly associated with worse sexual and hormonal functions. No relationship between adrenal androgen levels and these functions has been reported. Recently, a new class of CYP17 inhibitor, abiraterone acetate, was approved for treating castration-resistant prostate cancer [19]. Abiraterone acetate significantly reduced the serum adrenal and testicular androgen levels [20]. Further analyses focusing on sexual and hormonal functions are warranted in patients treated with new hormone agents.

This study had several limitations. The first is the small number of patients subjected to the multivariate analysis. Another is the evaluation of cognitive function. We used only MMSE scores, and this score does not cover details of cognitive functions. However, this is the first study to examine the association of testicular and adrenal androgen levels and ADT with cognitive and sexual/hormonal functions. Further large-scale studies are warranted.

\section{Conclusion}

LH-RH agonist monotherapy worsened sexual and hormonal functioning and hormonal bother, but not sexual bother or cognitive function, as assessed by the MMSE and EPIC questionnaires. The observed changes were associated with adrenal and residual testicular androgen levels.

\section{Competing interests}

K.S. and K.I. received honoraria from Takeda Pharmaceutical and K.S. received research grants from Takeda Pharmaceutical.

\section{Authors' contributions}

$\mathrm{KO}$ and $\mathrm{KS}$ designed the research; $\mathrm{YS}, \mathrm{MN}, \mathrm{HK}$, and $\mathrm{HM}$ collected the data; $\mathrm{KO}, \mathrm{YS}, \mathrm{KI}$, and $\mathrm{KS}$ analyzed the data and wrote the draft; and YS, MN, HK, and HM contributed to the critical revisions. All of the authors approved the paper's submission and the final version of this article.

\section{Acknowledgments}

We thank Ms. Rie Suzuki for conducting MMSE evaluations.

Received: 2 January 2015 Accepted: 5 February 2015

Published online: 06 April 2015

\section{References}

1. Mohler JL, Kantoff PW, Armstrong AJ, Bahnson RR, Cohen M, D'Amico AV, et al. Prostate cancer, version 2.2014. J Natl Compr Canc Netw. 2014:12:686-718.

2. Higano CS. Side effects of androgen deprivation therapy: monitoring and minimizing toxicity. Urology. 2003;61 (2 Suppl 1):32-8.

3. Ahmadi H, Daneshmand S. Androgen deprivation therapy: evidence-based management of side effects. BJU Int. 2013;111:543-8.

4. Nishii M, Nomura M, Sekine Y, Hoike H, Matsui H, Shibata Y, et al. Luteinizing hormone $(\mathrm{LH})$-releasing hormone agonist reduces serum adrenal androgen levels in prostate cancer patients: implications for the effect of LH on the adrenal glands. J Androl. 2012;33:1233-8.

5. Kakehi Y, Takegami M, Suzukamo Y, Namiki S, Arai Y, Kamoto T, et al. Health related quality of life in Japanese men with localized prostate cancer treated with current multiple modalities assessed by a newly developed Japanese version of the expanded prostate cancer index composite. J Urol. 2007:177:1856-61.

6. Wei JT, Dunn RL, Litwin MS, Sandler HM, Sanda MG. Development and validation of the expanded prostate cancer index composite (EPIC) for comprehensive assessment of health-related quality of life in men with prostate cancer. Urology. 2000;56:899-905.

7. Tombaugh TN, Mclntyre NJ. The mini-mental state examination: a comprehensive review. J Am Geriatr Soc. 1992:40:922-35.

8. Yamanaka H, Ito K, Naito S, Tsukamoto T, Usami M, Fujimoto H, et al. Effectiveness of adjuvant intermittent endocrine therapy following neoadjuvant endocrine therapy and external radiation therapy in men with locally advanced prostate cancer. Prostate. 2005;63:56-64.

9. Nelson CJ, Lee JS, Gamboa MC, Roth AJ. Cognitive effects of hormone therapy in men with prostate cancer. Cancer. 2008;113:1097-106.

10. Alibhai SMH, Breunis H, Timilshina N, Marzouk S, Stewart D, Tannock I, et al. Impact of androgen-deprivation therapy on cognitive function in men with nonmetastatic prostate cancer. J Clin Oncol. 2010;28:5030-7.

11. Mohile SG, Lacy M, Rodin M, Bylow K, Dale W, Meager MR, et al. Cognitive effects of androgen deprivation therapy in an old cohort of men with prostate cancer. Crit Rec Oncol Hematol. 2010;75:152-9.

12. Chao HH, Uchio E, Zhang S, Hu S, Bednarski SR, Luo X, et al. Effects of androgen deprivation on brain function in prostate cancer patients-a prospective observational cohort analysis. BMC Cancer. 2012;12:371-8.

13. Lee H, McGovern K, Finkelstein JS, Smith MR. Changes in bone mineral density and body composition during initial and long-term gonadotropin-releasing hormone agonist treatment for prostate carcinoma. Cancer. 2005;104:1633-7.

14. Gay HA, Michalski JM, Hamstra DA, Wei JT, Dunn RL, Klein EA, et al. Neoadjuvant androgen deprivation therapy leads to immediate impairment of vitality/hormonal and sexual quality of life: results of a multicenter prospective study. Urology. 2013;82:1363-9.

15. Yeap BB. Hormonal changes and their impact on cognitive and mental health of ageing men. Maturitas. 2014;79:227-35.

16. Byers AL, Yaffe K. Depression and risk of developing dementia. Nat Rev Neurol. 2011;7:323-31.

17. Salminen EK, Portin Rl, Koskinen Al, Helenius HY, Nurmi MJ. Estradiol and cognition during androgen deprivation in men with prostate carcinoma. Cancer. 2005;103:1381-7.

18. Lakshman KM, Kaplan B, Travison TG, Basaria S, Knapp PE, Singh AB, et al. The effects of injected testosterone dose and age on the conversion of testosterone to estradiol and dihydrotestosterone in young and older men. J Clin Endocrinol Metab. 2010;95:3955-64. 
19. de Bono JS, Logothetis CJ, Molina A, Fizazi K, North S, Chu L, et al. Abiraterone and increased survival in metastatic prostate cancer. N Engl J Med. 2011;364:1995-2005.

20. Attard G, Reid AH, Yap TA, Raynaud F, Dowsett M, Settatree S, et al. Phase I clinical trial of a selective inhibitor of CYP17, abiraterone acetate, confirms that castration-resistant prostate cancer commonly remains hormone driven. J Clin Oncol. 2008;26:4563-71.

Submit your next manuscript to BioMed Central and take full advantage of:

- Convenient online submission

- Thorough peer review

- No space constraints or color figure charges

- Immediate publication on acceptance

- Inclusion in PubMed, CAS, Scopus and Google Scholar

- Research which is freely available for redistribution 